\title{
LETTERS
}

\section{Availability of medical assistance in dying can be therapeutic}

The legalization of medical assistance in dying (MAiD) in Canada has created many logistical challenges for institutions and health care providers. In a country where diversity is not merely supported, but encouraged, it is not surprising that there are many outspoken critics of MAiD. One of us (I.M.B.) is the medical chair of our hospital MAiD committee; the other (S.A.) acts as a MAiD provider. Having been involved in over 30 referrals since June 2016, we have several important observations to make.

The gratitude expressed to us by families and patients is staggering. A recurring theme is that patients' moods are tremendously improved with the knowledge that MAiD gives them control over their disease. In some completed cases, symptoms were not controlled to an acceptable degree by aggressive palliative care. In some other cases, despite excellent symptom control with palliative care, patients desired control over the circumstances and timing of their deaths, and so chose MAiD. As per the law, all MAiD patients' natural deaths were reasonably forseeable. For patients who received MAiD, being able to prepare for their deaths, assemble their families, and die in a comfortable, dignified manner, were the universally espoused virtues of MAiD.

We have found that MAiD deaths provide a greater level of patient comfort than even the deaths from the withdrawal of life support in intensive care units. MAiD allows the use of intravenous medications in anesthetic doses, combined with neuromuscular blockade. At our institution, the procedure lasts only a few minutes, and avoids the dyspnea and increased work of breathing, which is so often associated with even the best palliative care or withdrawal of life support.

Although we support palliative care and believe it to be the right experience for most individuals, we have witnessed cases where palliative care was insufficient to manage the degree of suffering, or where patients simply wanted to avoid perceived indignities and loss of control associated with their progressive diseases. It has impressed us tremendously that the availability of MAiD has improved the outlook of many patients who have not chosen the procedure. The knowledge that MAiD is an option, should symptoms become unbearable, is very reassuring for patients and their families.

\section{Ian M. Ball MD MSc}

Division of Critical Care Medicine, Department of Epidemiology and Biostatistics, Western University Medical, and Chair, London Health Sciences Center MAiD Internal Review Committee

\section{Scott Anderson MD}

Divisions of Emergency and Critical Care Medicine, MAiD Provider, London Health Sciences Center, London, Ont.

— Cite as: CMAJ 2017 March 6;189:E372. doi: $10.1503 / \mathrm{cmaj} .732632$

\section{Reference}

1. Sibbald B. Uncertainty over MAiD likely to continue. CMAJ 2016;188:E417-18.

Competing interests: See affiliations. 